\title{
GTF Transverse and Longitudinal Emittance Data Analysis Technique*
}

\author{
J.F. Schmerge, J.E. Clendenin, D.H. Dowell and S.M. Gierman
}

\begin{abstract}
The SSRL Gun Test Facility (GTF) was built to develop a high brightness electron injector for the LCLS and has been operational since 1996. Measurements at the GTF include quadrupole scan transverse emittance measurements and linac phase scan longitudinal emittance measurements. Typically the beam size is measured on a screen as a function of a quadrupole current or linac phase and the beam matrix is then fit to the measured data. Often the emittance which is the final result of the measurement is the only number reported. However, the method used to reduce the data to the final emittance value can have a significant effect on the result. This paper describes in painful detail the methods used to analyze the transverse and longitudinal emittance data collected at the GTF.
\end{abstract}

\section{Introduction}

The Gun Test Facility (GTF) at SLAC was constructed in 1996 to produce a high brightness electron beam capable of driving the Linac Coherent Light Source. The GTF consists of a Nd:glass laser, 1.6 cell rf gun, emittance compensating solenoid, $3 \mathrm{~m}$ SLAC linac section, quadrupole doublet, multiple electron beam screens and spectrometer magnet with a vertical bend. The layout is shown in Figure 1. This paper describes transverse and longitudinal emittance measurements performed at the GTF using three different electron beam screens at different longitudinal positions. A detailed description of the data analysis techniques is also included. Identical analysis was used on simulated images from the beam simulation code PARMELA with a small amount of noise added. This data was analyzed for and presented at the X-ray FEL Commissioning Workshop held at DESY Zeuthen in April 2005 so that is could be compared with alternative data analysis techniques.

Emittance measurements at the GTF are composed of four main steps listed below:

- Image Acquisition

- Compute Beam Size from Images

- Beam Transport Model

- Least Square Fit

Image acquisition is described later in this section. The steps required to reduce the images to a beam size are described in section 2 along with the specific algorithm used for GTF image analysis. The beam transport model and the least square fit used to determine the beam matrix and other fit parameters is described in section 4 .

\footnotetext{
* Work supported in part by the DOE Contract DE-AC02-76SF00515.

This work was performed in support of the LCLS project at SLAC
} 


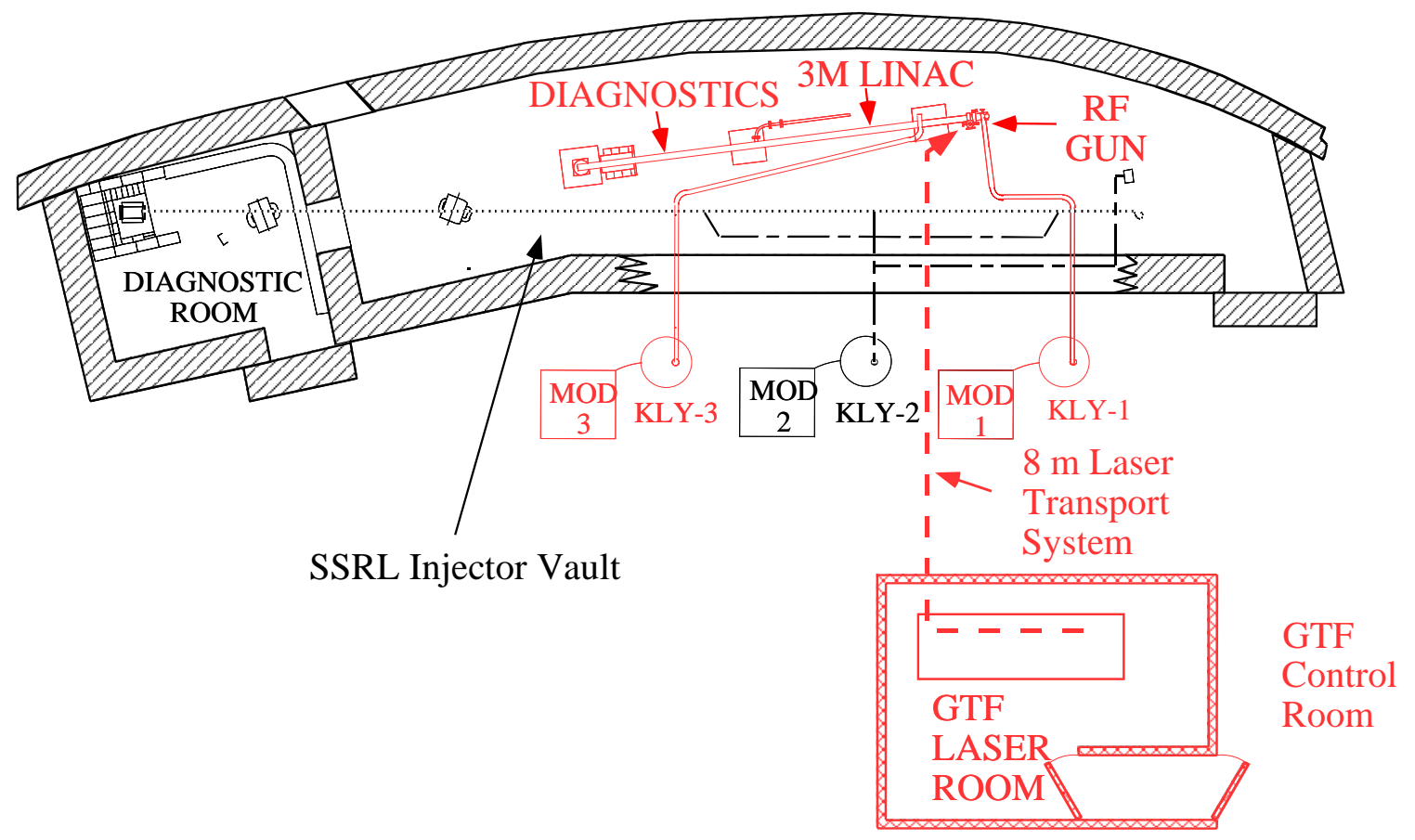

Figure 1: The Gun Test Facility at SLAC.

The gun is a 1.6 cell, S-band rf gun with $\mathrm{Cu}$ cathode as shown in Figure 2. The electrons exit the gun at roughly $5 \mathrm{MeV}$ and are accelerated to approximately $30 \mathrm{MeV}$ through the linac and then enter the diagnostic section. The quadrupole doublet is used to focus the beam onto the screens and also for quadrupole scan emittance measurements.

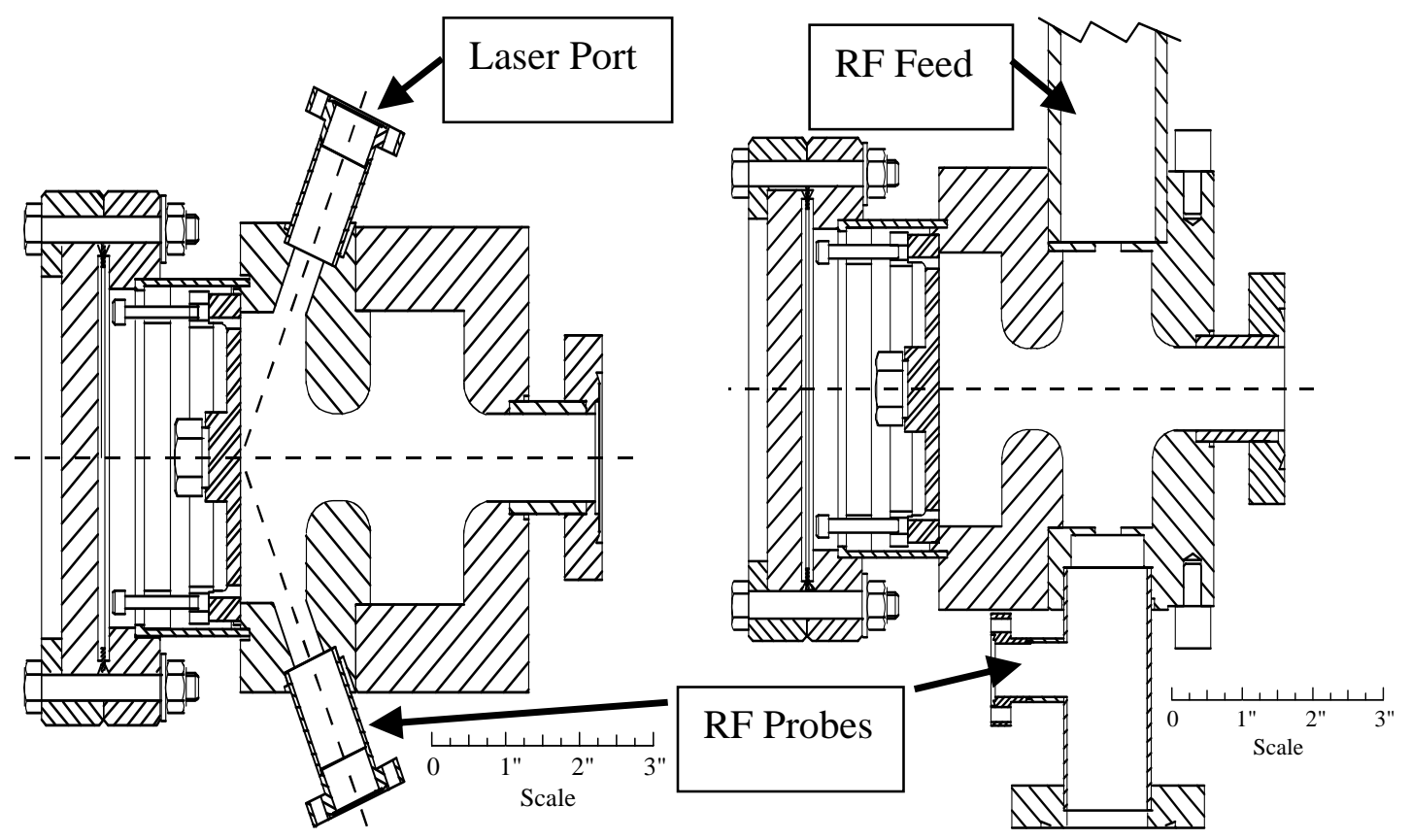

Figure 2: Two views of the GTF rf gun are shown. The figure on the right is rotated 45 degrees about the beam axis. The cathode is on the left and the beam exits on the right. 
The horizontal beam size was measured on all three screens as a function of quadrupole strength for horizontal emittance measurements. Temporally resolved emittance measurements were obtained by operating the linac sufficiently off crest to produce a linear energy chirp along the bunch and measuring the beam size on the phosphor screen in the dispersive region. In addition to the temporally resolved beam matrix, the slice offset angle and position can also determined as will be shown below.

Longitudinal emittance measurements were conducted by measuring the vertical beam size on the phosphor screen as a function of linac phase. As part of the longitudinal emittance measurement the beam energy is measured and the linac accelerating voltage and phase is fit to the data as well as the longitudinal beam matrix.

Electron beam images were captured on three separate screens downstream of the quadrupoles. The first is an OTR screen composed of a thin Aluminum foil stretched over a frame and inserted at a 45 degree angle with respect to the beam. The second screen is $100 \mu \mathrm{m}$ thick, $10 \mathrm{~mm}$ diameter YAG screen inserted at normal incidence with respect to the beam. A polished metal mirror roughly $1 \mathrm{~cm}$ downstream of the screen reflects the light out of the vacuum pipe, through a quartz window and into a camera. The third screen is labeled the spectrometer screen is mounted at 45 degrees with respect to the beam axis downstream of the spectrometer magnet in a dispersive region. The screen was manufactured at SLAC by depositing phosphor on a thin Aluminum substrate.

Analog cameras with $640 \mathrm{X} 480$ pixels were used with an 8 bit frame grabber to collect beam images for emittance measurements. A gated, intensified camera manufactured by Xybion was used to acquire images from the OTR screen due to the low signal levels from the OTR screen. The gating time was approximately 10-20 ns to reduce the dark current signal. Different optics on all three screens were used obtain the correct field of view for the particular screen. The calibration or each camera was measured by collecting images of a ruler on the bench using the identical optics and frame grabber. All the optics were locked down and the camera moved to the beamline and the position adjusted to bring the screen image into focus. The calibration for each camera/screen is listed in Table 1.

Table 1: GTF camera calibrations.

\begin{tabular}{|l|c|}
\hline Screen & Calibration $(\mu \mathrm{m} /$ pixel $)$ \\
\hline OTR & 3.99 \\
\hline YAG & 14.8 \\
\hline Spectrometer & 42.0 \\
\hline
\end{tabular}

The frame grabber black and white levels are adjusted to prevent the frame grabber from saturating. The frame grabber is adjusted until the maximum pixel value is $<255$ by looking at a histogram of the pixel values in an image. A variable attenuator is used to adjust the light level in the camera to avoid camera saturation. Two polarizers, one on a rotational stage, are mounted between the camera lens and vacuum window. One polarizer is rotated as the quadrupole or linac phase is adjusted to keep the maximum pixel level approximately constant.

All the currents in the magnets and various rf field amplitudes and phases are recorded for the same shot as the images. These values are used in the beamline model to 
determine the beam matrix. The charge is also recorded for each shot and is measured on toroids located before and after the linac. The toroids are calibrated against independent Faraday cups located before the linac and downstream of the spectrometer.

\section{GTF Image Analysis Technique}

The computation of electron beam sizes is a three step process listed below:

- Background Subtraction

- Define the Region of Interest (ROI)

- Calculate Beam Size

Background subtraction is necessary to remove dark current generated at the cathode by field emission. The background subtraction also reduces any effect from ambient light or systematic camera errors such as radiation damaged pixels and baselines. In order to eliminate the dark current, background images are obtained by eliminating the drive laser pulse. At the GTF, the laser operates at $2.5 \mathrm{~Hz}$ and the accelerator run at 5 or $10 \mathrm{~Hz}$. Thus background images are obtained in between laser shots. For the data reported here a single background image was obtained for each quadrupole current or linac phase setting. With a single background image the random noise is increased by a factor of $\sqrt{2}$. Of course this can be reduced with multiple background images. Typical background and beam images on the three screens are shown in Figure 3. No attempt is made to compensate for the shot to shot fluctuations in the background image which is most prevalent in the OTR screen images. The noise in the OTR screens is dominated by electrical noise from the fast gating electronics.

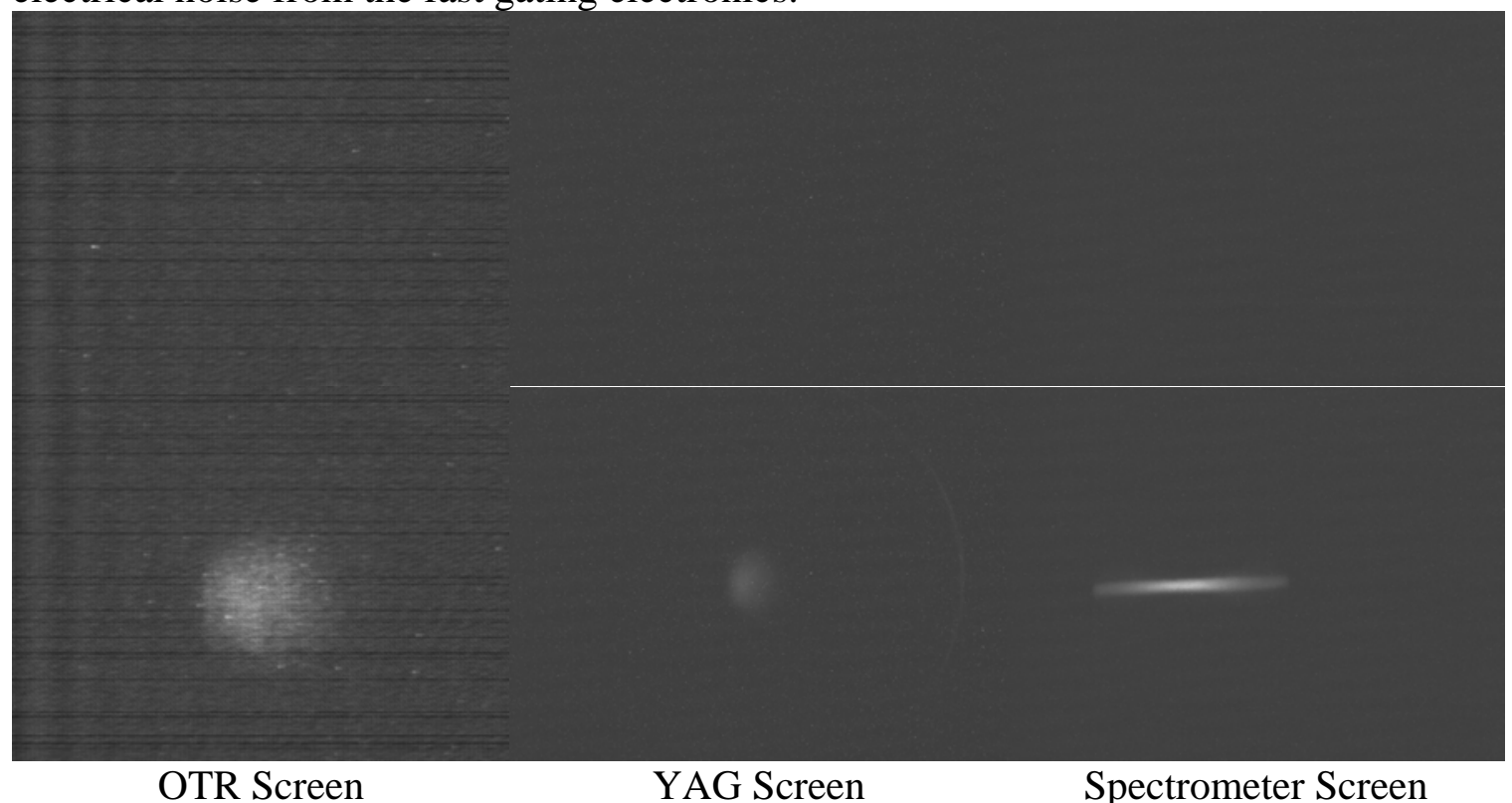

Figure 3: The OTR, YAG and spectrometer screen images are shown left to right. A background image is shown on top and a beam image on the bottom. 
After background subtraction a ROI is defined. The purpose of the ROI is to eliminate pixels that contribute only noise to the beam size computation and to limit the cumulative error caused by small offsets in the baseline.

The ROI used at the GTF is rectangular box an integral number of pixels long and wide. The dimensions of the ROI are determined by calculating the point where the horizontal and vertical projections fall below a specified fraction of the peak value. A new ROI is then computed using a projection over the old ROI and the process iterated until the ROI no longer changes. This typically requires only 2 or 3 iterations. The threshold level used for the published GTF data is 5\%. Several beam images with the rectangular ROI border plotted in red are shown in Figure 4 along with the vertical projection used to determine the horizontal beam size.

The algorithm described above does not always select an ROI that completely surrounds electron beam. For example, a large tail or halo on the beam can easily be clipped. Also, a bright pixel or group of pixels separate from the beam can sometimes be selected instead of the beam. Thus it is preferred that the ROI is visually verified to properly surround the electron beam before the computed electron beam size is used in the determination of the beam matrix.
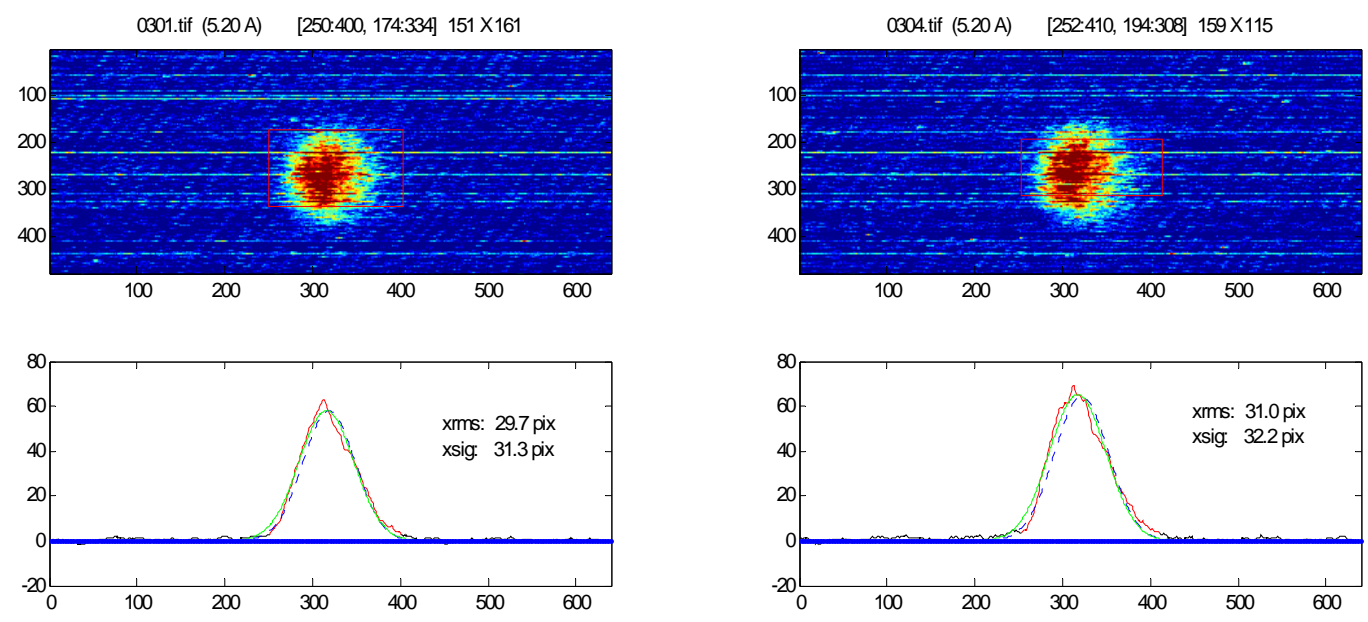

Figure 4: Two beam images from the OTR screen are shown on top with the computed ROI plotted as a red rectangle and the pixel coordinates of the ROI are shown. The vertical projection over the ROI is plotted as a red line and a Gaussian fit as a dashed line below the image. The rms beam size and standard deviation from the Gaussian fit are also shown.

The temporally resolved emittance is obtained by slicing a chirped beam image into multiple beamlets. A spectrometer screen image is shown in Figure 5 with the projected image ROI plotted in red and the ROI of each slice in yellow. Each of the 10 slice ROI's width is equal to $10 \%$ of the width of the projected ROI. The height of the slice ROI's is determined using the same algorithm described above. The energy or time axis is horizontal in the image and the horizontal beam size is on the image's vertical axis. 


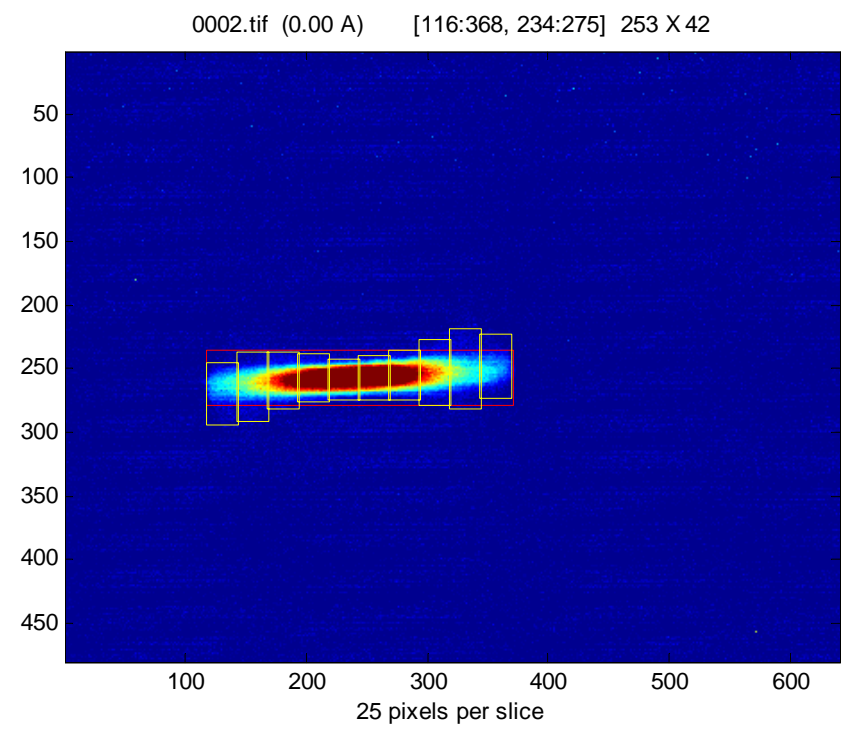

Figure 5: A spectrometer screen image is shown with the projected beam ROI and the ROI for 10 slices. The projected beam is 253 pixels wide by 42 pixels high. Each slice is 25 pixels wide but with different heights as determined by the ROI algorithm.

The beam size is then calculated from the pixel values distribution, $f\left(x_{i}, y_{j}\right)$, inside the ROI where $\mathrm{i}$ and $\mathrm{j}$ are the pixel coordinates. Both the rms beam size and the standard deviation using a Gaussian fit are calculated. The formula for the rms width is shown in equation 1 with the centroid $x_{c}$ defined in equation 2. The Gaussian fit is determined by minimizing the error function defined in equation 3 where $A_{G}$ is the amplitude and $\Delta x_{G}$ and $\mathrm{x}_{\mathrm{cG}}$ are the Gaussian width and centroid respectively.

$$
\begin{aligned}
\Delta x & =\sqrt{\frac{\sum_{i}^{R O I}\left(x_{i}-x_{c}\right)^{2} \sum_{j}^{R O I} f\left(x_{i}, y_{j}\right)}{\sum_{i}^{R O I} \sum_{j}^{R O I} f\left(x_{i}, y_{j}\right)}} \\
x_{c} & =\frac{\sum_{i}^{R O I}\left(x_{i}-x_{c}\right) \sum_{j}^{R O I} f\left(x_{i}, y_{j}\right)}{\sum_{i}^{R O I} \sum_{j}^{R O I} f\left(x_{i}, y_{j}\right)} \\
\chi_{\text {Gaussian }}^{2} & =\sum_{i}^{R O I}\left(A_{G} e^{-\frac{\left(x_{i}-x_{c G}\right)^{2}}{2 \Delta x_{G}^{2}}}-\sum_{j}^{R O I} f\left(x_{i}, y_{j}\right)\right)^{2}
\end{aligned}
$$


The beam sizes computed from multiple images with identical beam parameters are averaged to compute the beam size used in the emittance fitting routine. The error in the beam size is the standard deviation from the multiple measurements. The data presented in this paper uses five beam images. The same background image is subtracted from all five beam images. The appendix lists the calculated beam size for the images used to compute emittances reported in this paper.

A comparison of rms and Gaussian widths, in pixels, is shown in Figure 6 as a function of threshold level for four different randomly chosen images. The Gaussian widths are relatively independent of threshold level since the fit is not weighted and thus dominantly fits the peak of the signal. The rms width can decrease as much as $20 \%$ as the threshold level increases from 1 to 15\%. Thus the Gaussian widths are more robust than the rms width calculation. However, many images, especially the longitudinal phase space data, show highly non-Gaussian distributions. Thus the rms beam size with a 5\% threshold is typically chosen for analyzing GTF data. All of the beam sizes reported here, except for those in Figure 6, were calculated with a 5\% threshold.
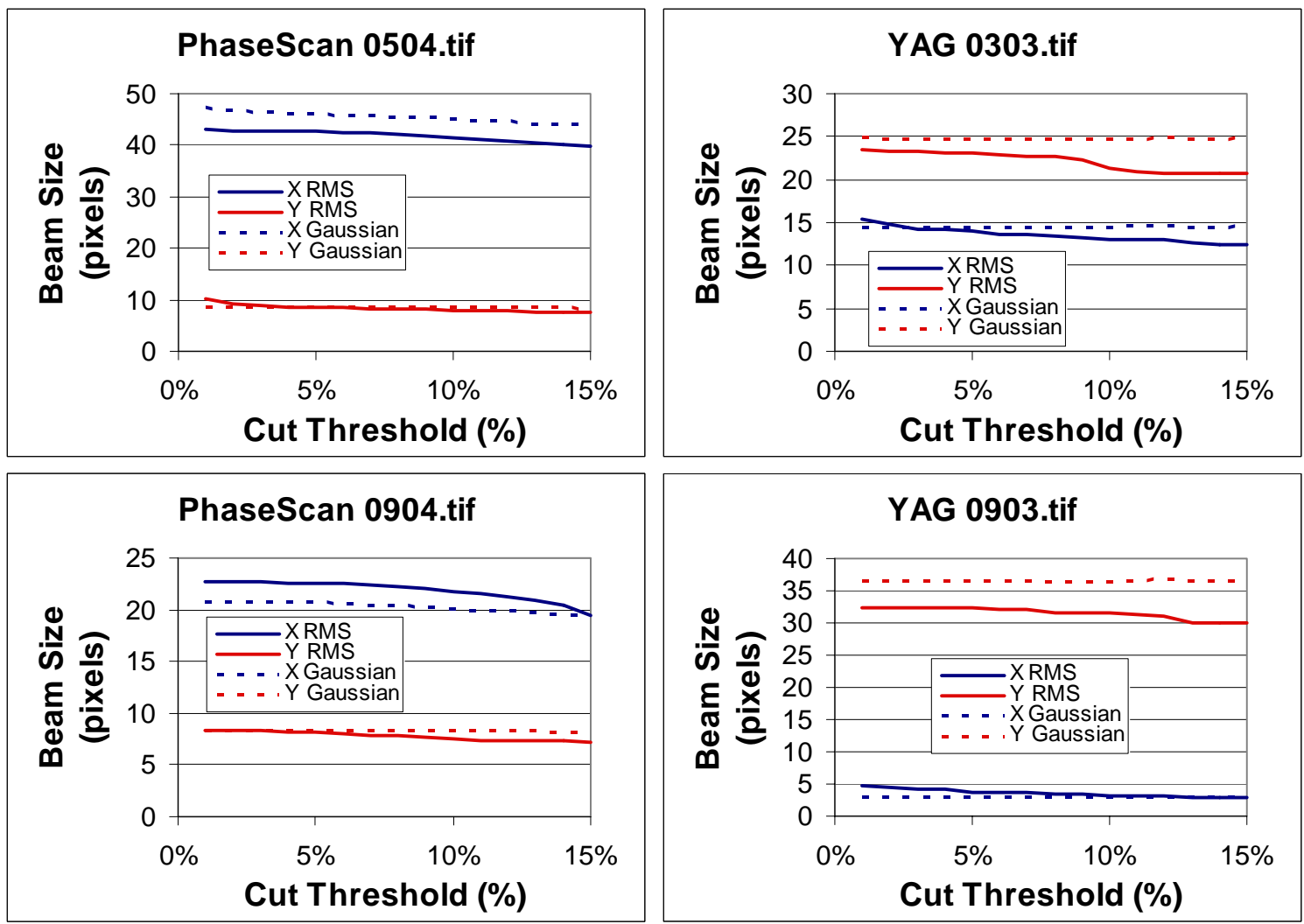

Figure 6: The $\mathrm{x}$ and $\mathrm{y}$ rms and Gaussian beam sizes as a function of ROI threshold level for four randomly chosen images are shown.

\section{Emittance Calculation Technique}

The computed beam sizes as a function of quadrupole current or linac phase are used to determine the transverse or longitudinal beam matrix. The three independent beam 
matrix parameters are fit by comparing the measured beam size to a mathematical model of the beamline. The beamline is modeled using the first order matrix presented in TRANSPORT [1].

The relevant beamline parameters are shown in Figure 7 including the quadrupole strength as a function of current, drift distances, and magnet lengths. The drift distance between magnets used in the Transport model is adjusted by the difference between the magnets physical length and the effective length so that the total distance in the matrix model matches the beamline distance. The first quadrupole focuses in the vertical plane and the second quadrupole focuses in the horizontal plane. The spectrometer magnet is a $60^{\circ}$ wedge magnet with the listed bending radius, pole face rotations, and effective fringe field pole face rotation.

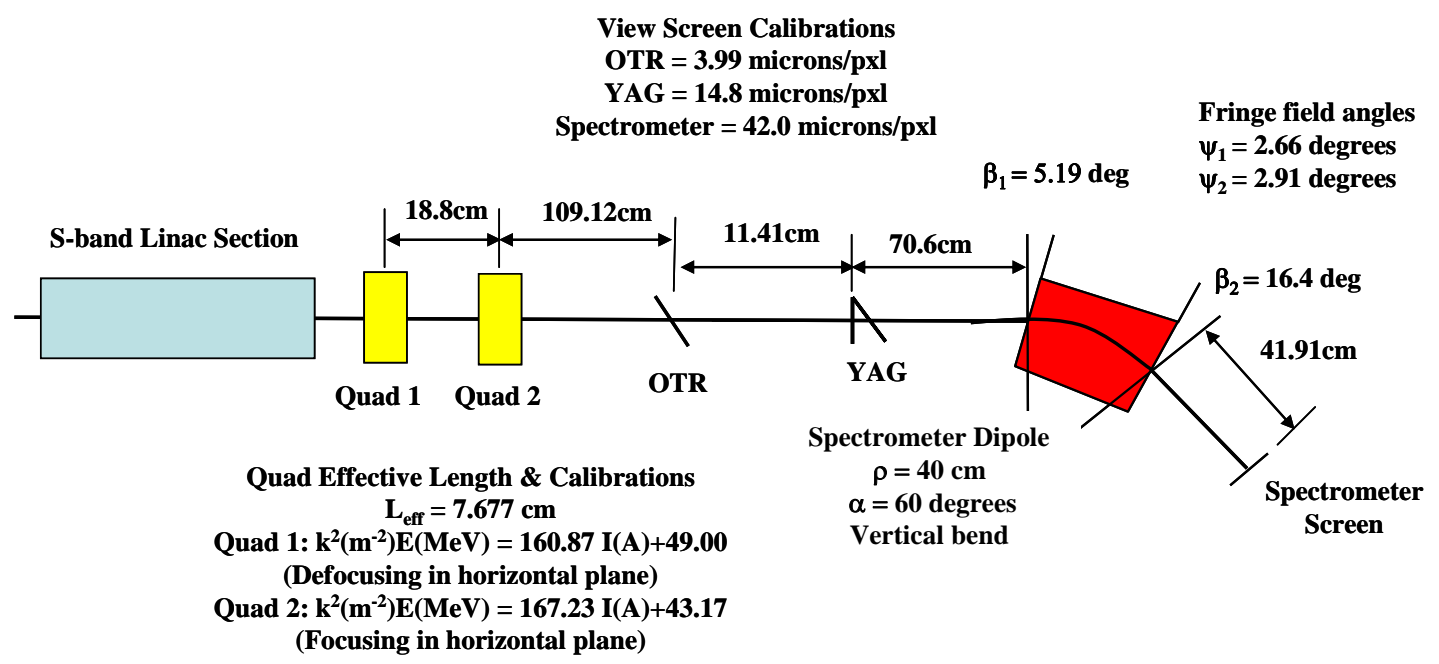

Figure 7: A block diagram of the GTF beamline with all the relevant beamline parameters.

The transverse beam matrix transforms along the beamline as shown in equation 4 where $\sigma_{11}, \sigma_{12}$, and $\sigma_{22}$ are the three beam matrix parameters at the initial point and $\sigma_{11 i}$, $\sigma_{12 \mathrm{i}}$, and $\sigma_{22 \mathrm{i}}$ are the identical parameters at the observation point. $\mathrm{A}_{\mathrm{i}}, \mathrm{B}_{\mathrm{i}}, \mathrm{C}_{\mathrm{i}}$, and $\mathrm{D}_{\mathrm{i}}$ are the total horizontal transport matrix coefficients from the starting point to the observation point and are functions of the quadrupole current. The total matrix is of course the product of the matrix for each individual element. The beam size of the $i^{\text {th }}$ observation can be written in terms of the transport matrix coefficients and the initial beam matrix as shown in equation 5.

$$
\begin{gathered}
{\left[\begin{array}{cc}
\sigma_{11 i} & \sigma_{12 i} \\
\sigma_{12 i} & \sigma_{22 i}
\end{array}\right]=\left[\begin{array}{cc}
A_{i} & B_{i} \\
C_{i} & D_{i}
\end{array}\right]\left[\begin{array}{cc}
\sigma_{11} & \sigma_{12} \\
\sigma_{12} & \sigma_{22}
\end{array}\right]\left[\begin{array}{ll}
A_{i} & B_{i} \\
C_{i} & D_{i}
\end{array}\right]^{T}} \\
\Delta x_{i}^{2} \equiv \sigma_{11 i}=A_{i}^{2} \sigma_{11}+2 A_{i} B_{i} \sigma_{12}+B_{i}^{2} \sigma_{22}
\end{gathered}
$$

The final step is to fit the beam matrix parameters to minimize the error between the measured beam size and the beam size computed using the model. However, the error can be defined in multiple ways. Two definitions of the error function are listed in 
equations 6 and 7 . The first is the so called linear definition since the derivative of the error function is a linear function of the beam matrix parameters. The beam matrix parameters that minimize the linear error function can be determined by inverting a matrix. However, this error function is fitting the square of the measured beam size instead of just the beam size. The non-linear error function fits the beam matrix parameters to the measured beam size but unfortunately no analytic solution exists to minimize the error function. Instead, this error function must be minimized numerically. The published GTF data as well as the data presented here are fit with the non-linear error function since the beam size and not the beam size squared is the quantity physically measured. The beam matrix parameters from the linear fitting are used as a seed for the non-linear fit. Typically the beam matrix parameters from the non-linear fit are only a few percent different from the linear fit.

$$
\begin{aligned}
& \chi_{\text {linear emittance }}^{2}=\sum_{i=1}^{N}\left(\frac{\Delta x_{\text {ibeam fit }}^{2}-\Delta x_{\text {ibeam measure }}^{2}}{2 \Delta x_{\text {ibeam measure }} \sigma_{\Delta x_{\text {ibeam measure }}}}\right)^{2}=\sum_{i=1}^{N}\left(\frac{A_{i}^{2} \sigma_{11}+2 A_{i} B_{i} \sigma_{12}+B_{i}^{2} \sigma_{22}-\Delta x_{\text {ibeam measure }}^{2}}{2 \Delta x_{\text {ibeam measure }} \sigma_{\Delta x_{\text {ibeam measure }}}}\right)^{2} 6 \\
& \chi_{\text {non-linear emittance }}^{2}=\sum_{i=1}^{N}\left(\frac{\Delta x_{i \text { ibeam fit }}-\Delta x_{\text {ibeam measure }}}{\sigma_{\Delta x_{\text {ibeam measure }}}}\right)^{2}=\sum_{i=1}^{N}\left(\frac{\sqrt{A_{i}^{2} \sigma_{11}+2 A_{i} B_{i} \sigma_{12}+B_{i}^{2} \sigma_{22}}-\Delta x_{\text {ibeam measure }}}{\sigma_{\Delta x_{\text {ibeam measure }}}}\right)^{2} 7
\end{aligned}
$$

With a chirped beam on the spectrometer screen it is evident that the beam has a position-time correlation or tilt. Thus in addition to fitting the beam matrix, the centroid position and angle can be fit for each slice. The centroid of each slice relative to the projected beam centroid is measured at the spectrometer screen. The error function is defined in equation 8 where $\mathrm{x}_{0}$ and $\mathrm{x}_{0}$ ' are the position and angle of the slice at the initial longitudinal point.

$$
\chi_{\text {centroid }}^{2}=\sum_{i=1}^{N}\left(\frac{x_{i \text { fit }}-x_{\text {imeasure }}}{\sigma_{x_{\text {imeasure }}}}\right)^{2}=\sum_{i=1}^{N}\left(\frac{A_{i} x_{0}+B_{i} x_{0}^{\prime}-x_{\text {imeasure }}}{\sigma_{x_{\text {imeasure }}}}\right)^{2}
$$

The transport matrix used for the longitudinal measurements begins at the linac and ends at the spectrometer screen. The beam is assumed to travel at the speed of light and therefore there is no phase slippage. This assumption is not valid in the linac where the beam enters at only $5 \mathrm{MeV}$ with a large energy spread. However, since all the particles slip similar amounts the net result is a small change in the definition of linac phase and the non-linear effect is ignored in the emittance analysis. Analogous to equation 5, the energy spread at the spectrometer screen can be written in terms of the longitudinal transport matrix coefficients, $\mathrm{C}_{\mathrm{li}}$ and $\mathrm{D}_{\mathrm{li}}$, and the longitudinal beam matrix at the entrance to the linac as shown in equation 9.

$$
\Delta E_{i}^{2} \equiv \tau_{22 i}=C_{l i}^{2} \tau_{11}+2 C_{l i} D_{l i} \tau_{12}+D_{l i}^{2} \tau_{22}
$$


The beam energy is measured with the spectrometer magnet. From magnetic measurements, the beam energy is given in equation 10 as a function of spectrometer current and pixel location on the spectrometer screen. For the transverse emittance measurements the beam energy is constant and approximately $30 \mathrm{MeV}$. However, during the longitudinal measurement the energy varies sinusoidally as the linac phase is modulated. In this case the energy is fit to a sinusoid to determine the absolute linac phase and accelerating voltage. The error function is shown in equation 11 where the fit parameters are the linac voltage, $\mathrm{V}_{\text {linac }}$, and the phase offset, $\Delta \theta_{\text {linac. The voltage and }}$ phase are required to calculate the beam transport matrix coefficients and the phase is defined to be $0^{\circ}$ at the maximum energy.

$$
\begin{aligned}
& E(\mathrm{MeV})=\left(-1.421^{-5} I_{\text {spect }}^{3}+1.204^{-3} I_{\text {spect }}^{2}+0.9992 I_{\text {spect }}+0.5541\right)\left(1+0.0000724\left(348-p_{\text {centroid }}\right)\right) \\
& \chi_{\text {energy }}^{2}=\sum_{i=1}^{N}\left[\frac{E_{\text {ifit }}-E_{\text {imeasure }}}{\sigma_{E_{\text {imeasure }}}}\right]^{2}=\sum_{i=1}^{N}\left[\frac{\left(E_{\text {gun }}+V_{\text {linac }} \cos \left(\theta_{\text {ilinac }}+\Delta \theta_{\text {linac }}\right)\right)-E_{\text {imeasure }}}{\sigma_{E_{\text {imeasure }}}}\right]^{2} 11
\end{aligned}
$$

\section{Experimental Results}

The longitudinal emittance results are shown in Figure 8 which shows the measured energy and energy spread versus the linac phase along with the fits and the phase space ellipses at three positions along the beamline.. The linac phase is defined as $0^{\circ}$ at the maximum energy. The model is in excellent agreement with the measured data. Table 2 shows the longitudinal beam parameters at the linac entrance and exit with $\theta_{\text {linac }}=8^{\circ}$.
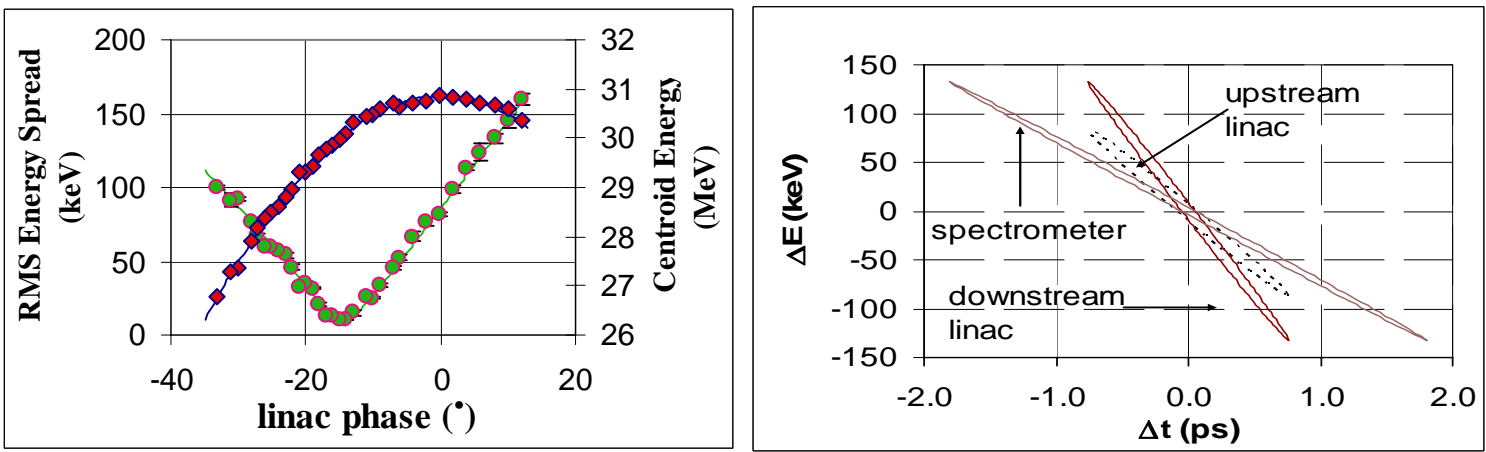

Figure 8: The measured energy spread in green and energy in red are plotted on the left as a function of the linac phase. The longitudinal, rms, phase space ellipse at the linac entrance, exit, and at the spectrometer screen is plotted on the right.

Table 2: The rms beam parameters at the linac entrance and exit are shown. The exit parameters are calculated with a linac phase of $8^{\circ}$ and gradient of $8.35 \mathrm{MV} / \mathrm{m}$.

\begin{tabular}{|l|c|c|c|}
\hline Beam Parameter & Linac Entrance & Linac Exit & Units \\
\hline$\tau_{11}$ & 0.575 & 0.575 & $\mathrm{ps}^{2}$ \\
\hline$\tau_{12}$ & -64.5 & -100 & $\mathrm{keV} \mathrm{ps}$ \\
\hline$\tau_{22}$ & 7310 & 17600 & $\mathrm{keV}^{2}$ \\
\hline
\end{tabular}


GTF Transverse and Longitudinal Emittance Data Analysis Technique

\begin{tabular}{|l|c|c|c|}
\hline$\varepsilon_{\mathrm{l}}$ & 6.84 & 6.84 & $\mathrm{keV} \mathrm{ps}$ \\
\hline$\varepsilon_{\mathrm{ln}}$ & 4.01 & 4.01 & $\mu \mathrm{m}$ \\
\hline$\sigma_{\mathrm{E}}$ & 85.5 & 133 & $\mathrm{keV}$ \\
\hline$\sigma_{\mathrm{E} \text { uncorrelated }}$ & 9.02 & 9.02 & $\mathrm{keV}$ \\
\hline$\sigma_{\mathrm{t}}$ & 0.758 & 0.758 & $\mathrm{ps}$ \\
\hline $\mathrm{dE} / \mathrm{dt}$ & -112 & -175 & $\mathrm{keV} / \mathrm{ps}$ \\
\hline
\end{tabular}

The longitudinal phase space is highly correlated at the linac entrance. The correlation can be removed after the linac when the phase is $-14^{\circ}$. Alternatively it can be increased if the linac phase is $>0^{\circ}$. The stronger correlations are used to conduct the temporally resolved transverse emittance measurements.

The sliced beam size measurements and centroid are plotted in Figure 9 along with the phase space ellipses. The centroids of each slice are measured with respect to the projected beam centroid. The Twiss parameters, offset angle, offset position and current vs time are shown in Figure 10. The measured Twiss parameters are plotted using both the Gaussian and rms beam sizes. The linac phase for these measurements was $8^{\circ}$ so the time axis is calibrated by measuring the beam energy and using the measured correlation listed in Table 2.
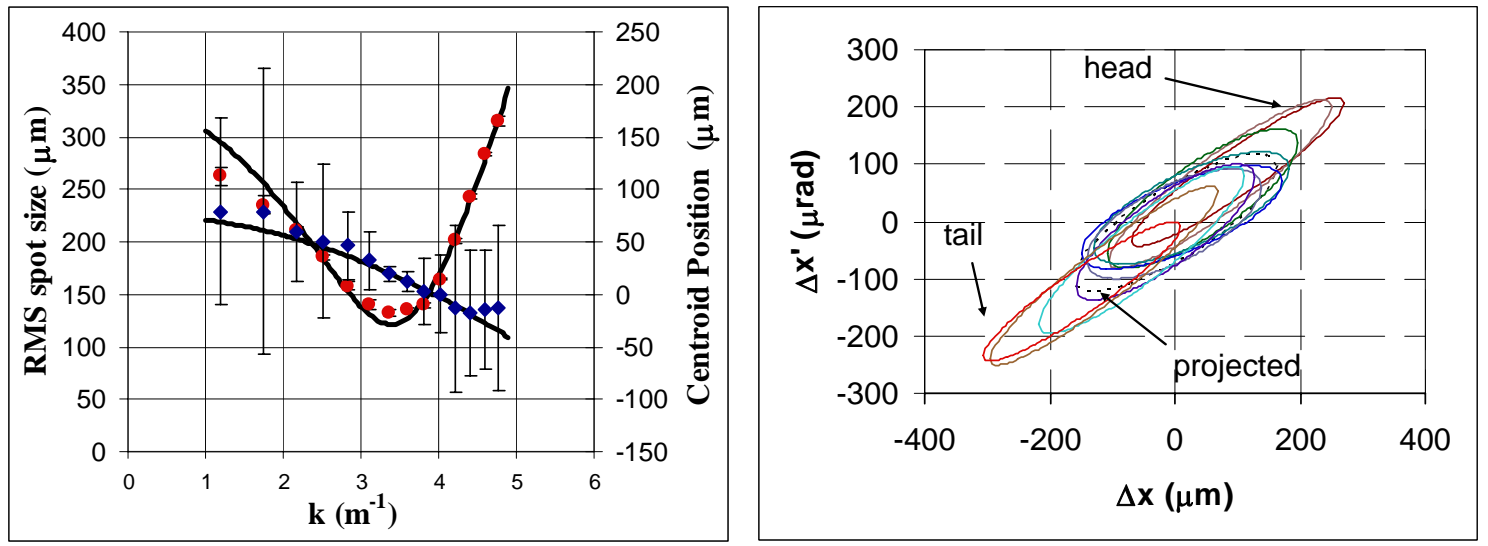

Figure 9: The measured beam size (red circles) and offset (blue diamonds) for slice number 4 are plotted on the left as a function of quadrupole strength along with the fits (solid lines). The phase space at the quadrupole for all 10 slices and the projected beam (dashed line) are shown on the right. The slice phase space ellipses are plotted using the measured angle and position offsets. 

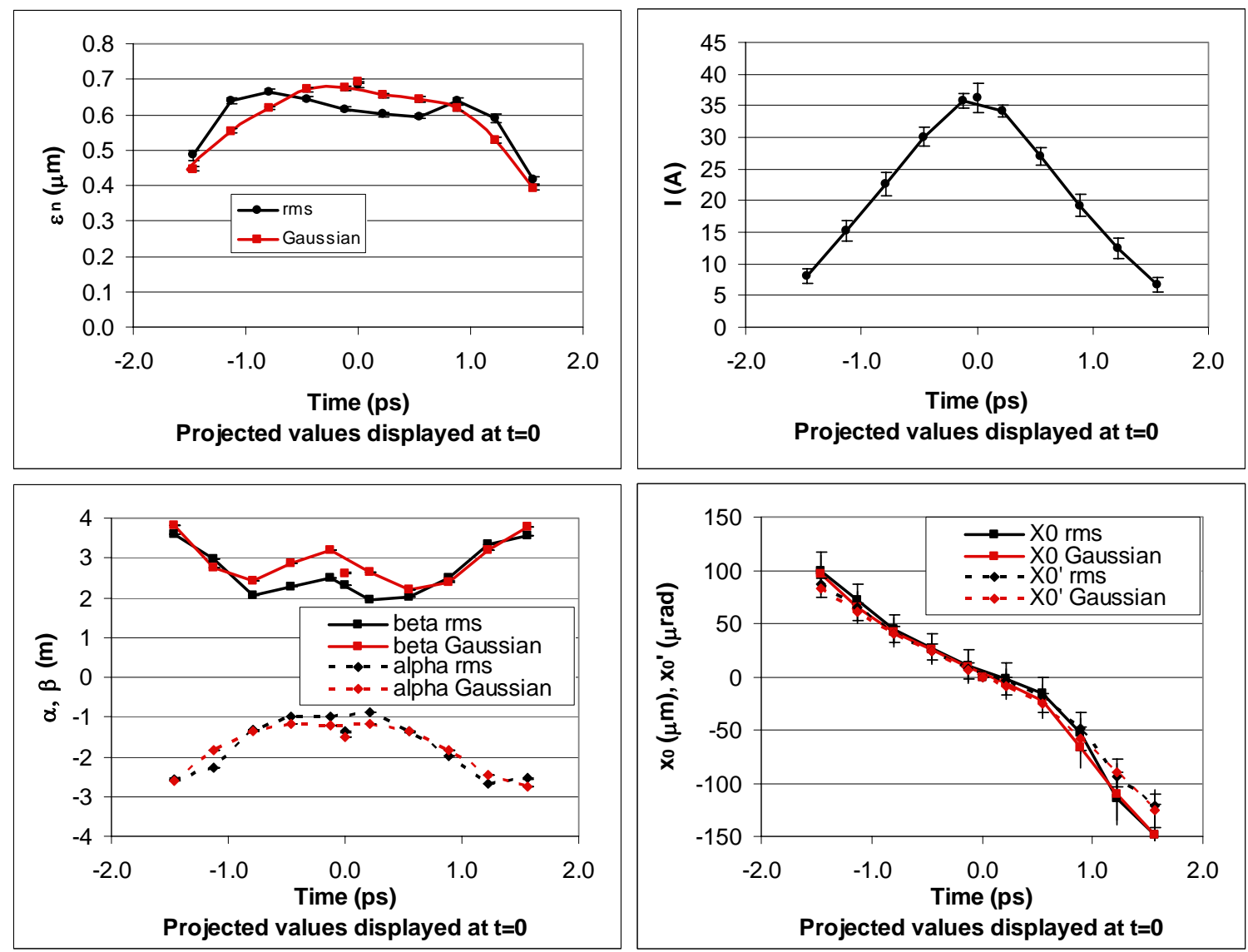

Figure 10: The emittance versus time is plotted in the upper left and the $\alpha$ and $\beta$ functions at the initial point are shown in the lower left. The current versus time is shown in the upper right based on the total charge and the charge contained in each slice. The measured offset angle and position at the initial point are shown in the lower right corner. The measurement of the projected beam is plotted at $t=0$ on all graphs.

The measured beam size versus quadrupole strength is shown in Figure 11 . The figure shows the projected beam size on all three screens and also the rms phase space ellipses at the entrance to quadrupole 1 from each screen measurement. There is good agreement among all three measurements. The OTR and YAG screens were measured with a linac phase of $16^{\circ}$ which is near the minimum energy spread while the spectrometer screen measurement was conducted at a linac phase of $8^{\circ}$ as described earlier. The beam parameters at the entrance to quadrupole 1 from all three measurements are included in Table 4. As expected the beam reaches a smaller minimum size and requires a shorter focal length the closer it is to the focusing quadrupole. 
GTF Transverse and Longitudinal Emittance Data Analysis Technique
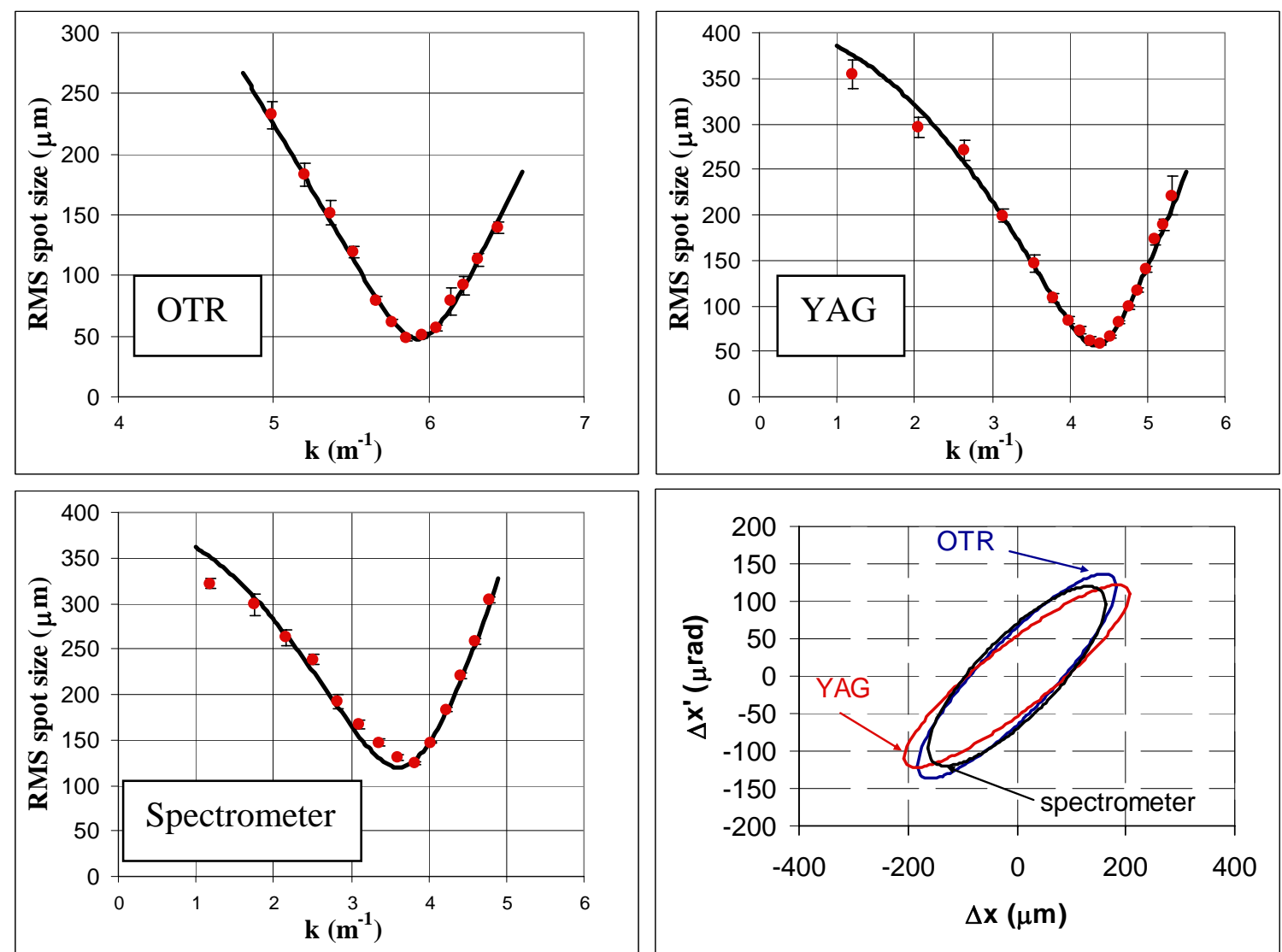

Figure 11: The measured, projected beam size versus quadrupole strength on all three screens. The rms phase space ellipses at the quadrupole are also shown.

Table 4: Beam parameters at the entrance to quadrupole 1 from the OTR, YAG and Spectrometer screen measurements.

\begin{tabular}{|l|c|c|c|c|}
\hline Beam Parameter & OTR & YAG & Spectrometer & Units \\
\hline$\sigma_{11}$ & 0.0335 & 0.0426 & 0.0264 & $\mathrm{~mm}^{2}$ \\
\hline$\sigma_{12}$ & 0.0220 & 0.0225 & 0.0157 & $\mu \mathrm{m}$ \\
\hline$\sigma_{22}$ & 0.0187 & 0.0148 & 0.0144 & $\mathrm{mrad}^{2}$ \\
\hline$\varepsilon_{\mathrm{g}}$ & 0.0120 & 0.0111 & 0.0114 & $\mu \mathrm{m}$ \\
\hline$\varepsilon_{\mathrm{n}}$ & 0.702 & 0.654 & 0.683 & $\mu \mathrm{m}$ \\
\hline$\sigma_{\mathrm{x}}$ & 183 & 206 & 162 & $\mu \mathrm{m}$ \\
\hline$\sigma_{\mathrm{x}}$ & 137 & 122 & 120 & $\mu \mathrm{rad}$ \\
\hline
\end{tabular}

\section{Analysis of Simulated Data}

In addition to using the measured electron beam images to calculate the beam matrix parameters, simulated images from PARMELA were used to test the analysis technique. The beam was simulated at three different longitudinal positions and then the data reduced to an image in $\mathrm{x}-\mathrm{y}$ space at each location. The PARMELA data was binned into 
"pixels" with various dimensions and some random noise added to the images. The noise had a Gaussian distribution and images with different average and rms noise values were generated. Two of the images from the simulated data are shown in Figure 12.

The calculated rms beam sizes using the image analysis software algorithm described earlier are then compared with PARMELA results computed from the 6-D coordinates of all the particles. Figure 13 shows the beam size on the three screens for three different beam parameters as well as the beam size calculated by PARMELA. The calculated emittance from each three screen test case and the pixel size and noise levels for each case are listed in Table 5. The emittance is calculated in identical manner as reported above except that the transport matrix is modified since the simulated data uses a three screen emittance measurement technique and the real measured data utilizes a quadrupole scan. In all cases the emittance is less than or equal to the emittance calculated by PARMELA and in almost all cases the beam size is smaller than computed by PARMELA. This is almost certainly due to the halo particles that are included in the PARMELA result but are filtered in the image analysis routine due to the $5 \%$ threshold limit.
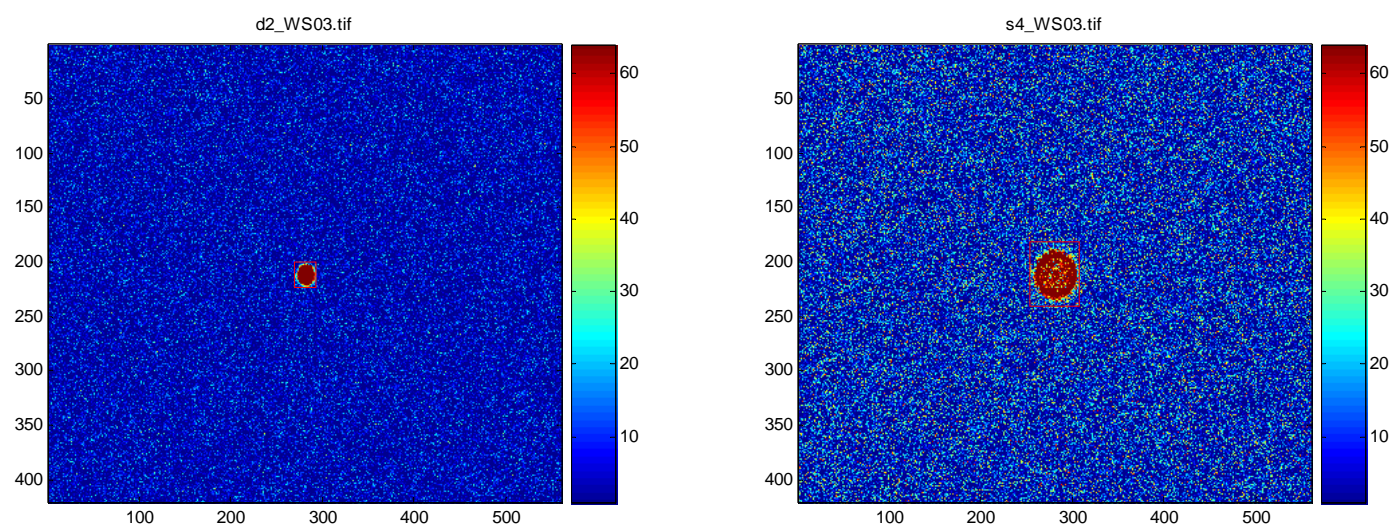

Figure 12: Two images at screen location three generated from the simulation code PARMELA. The image on the left has $20 \mu \mathrm{m}$ pixel size and 60 counts of average noise level with standard deviation of 10 . The image on the right has $10 \mu \mathrm{m}$ pixel size and 100 counts of average noise level with a standard deviation of 20. The red boxes are the ROI for each image as calculated using the algorithm described earlier.
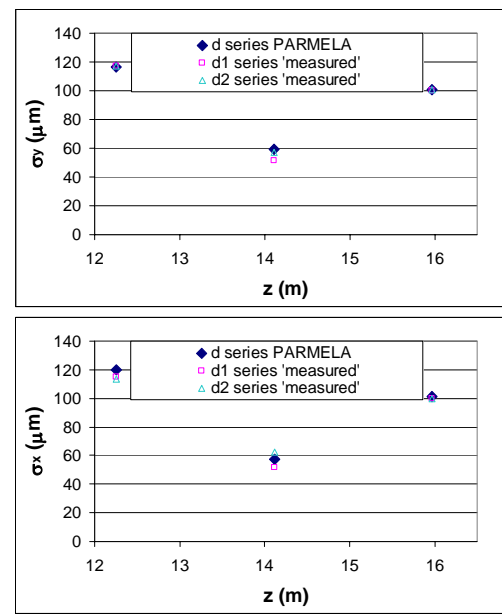
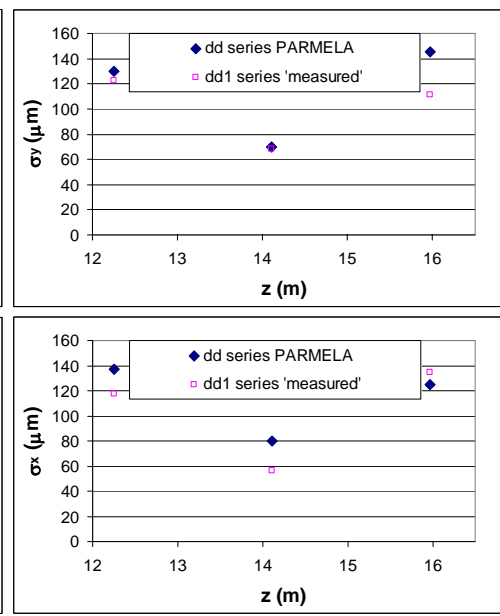
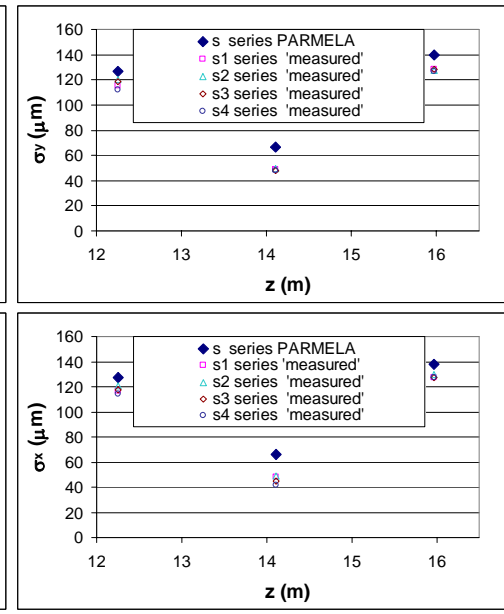
Figure 13: The horizontal rms beam size is shown on the bottom row and the vertical rms beam size on the top row for three different sets of beam parameters. Several different test cases for each set of beam parameters were run with different pixel size, average and standard deviation noise level.

Table 5: The horizontal and vertical emittance for the various simulated test cases.

\begin{tabular}{|l|c|c|c|c|c|}
\hline Case & $\varepsilon_{\mathrm{nx}}(\mu \mathrm{m})$ & $\varepsilon_{\mathrm{ny}}(\mu \mathrm{m})$ & Pixel $(\mu \mathrm{m})$ & Ave noise & Rms noise \\
\hline D (PARMELA) & 0.76 & 0.76 & NA & NA & NA \\
\hline D1 & 0.68 & 0.69 & 10 & 60 & 10 \\
\hline D2 & 0.76 & 0.74 & 20 & 60 & 10 \\
\hline DD (PARMELA) & 1.17 & 1.17 & NA & NA & NA \\
\hline DD1 & 0.90 & 0.91 & 10 & 60 & 10 \\
\hline S (PARMELA) & 1.07 & 1.08 & NA & NA & NA \\
\hline S1 & 0.76 & 0.77 & 10 & 60 & 10 \\
\hline S2 & 0.80 & 0.79 & 20 & 60 & 10 \\
\hline S3 & 0.72 & 0.77 & 10 & 60 & 20 \\
\hline S4 & 0.67 & 0.74 & 10 & 100 & 20 \\
\hline
\end{tabular}

\section{Conclusions}

As expected the data analysis revealed that the ROI affects the calculated beam size and thus the emittance. Different algorithms used to compute the ROI will produce different emittance results. The published GTF data uses a 5\% threshold limit to cut off the wings in an attempt to minimize this effect. Table 6 shows the measured projected emittance for each screen using both rms and Gaussian beam sizes. The Gaussian fits routinely produce a smaller minimum beam size and therefore lower emittance. The ratio of Gaussian to rms beam size also varies with the beam size as can be seen in the appendix. The Gaussian fit in the longitudinal data should not be trusted as the distribution is typically non-Gaussian. However, the Gaussian fits are less sensitive to the ROI.

Comparing the results of a simulated beam with PARMELA computations indicates the beam size and thus the emittance can be underestimated when compared with 100\% of the particles. This is not surprising since the data analysis necessarily excludes particles far from the centroid that are indistinguishable from noise and thus will compute a lower emittance.

Table 6: comparison of emittance measurements using rms and Gaussian beam sizes.

\begin{tabular}{|l|l|l|l|}
\hline Measurement & rms Fit & Gaussian Fit & Units \\
\hline OTR (Transverse) & $0.70 \pm 0.02$ & $0.61 \pm 0.01$ & $\mu \mathrm{m}$ \\
\hline YAG (Transverse) & $0.65 \pm 0.01$ & $0.61 \pm 0.01$ & $\mu \mathrm{m}$ \\
\hline Spectrometer (Transverse) & $0.68 \pm 0.01$ & $0.69 \pm 0.01$ & $\mu \mathrm{m}$ \\
\hline Spectrometer (Longitudinal) & $4.0 \pm 0.13$ & $1.9 \pm 0.42$ & $\mu \mathrm{m}$ \\
\hline
\end{tabular}

\section{Acknowledgments}


We would like to acknowledge the assistance from the rest of the GTF group including B. Murphy, J. Castro and P. Bolton. Without their help none of this work would have been possible. Also, C. Limborg provided all the simulated images for analysis as well as the PARMELA results. Finally we would like to acknowledge the useful discussions with $\mathrm{H}$. Loos regarding different methods for data analysis.

\section{Appendix}

The calculated beam sizes for the images used in the analysis in this paper. The table lists both the rms and Gaussian beam size for the real projected beam images in pixels and the $\mathrm{x}$ and $\mathrm{y}$ rms beam size for the simulated images. 
GTF Transverse and Longitudinal Emittance Data Analysis Technique
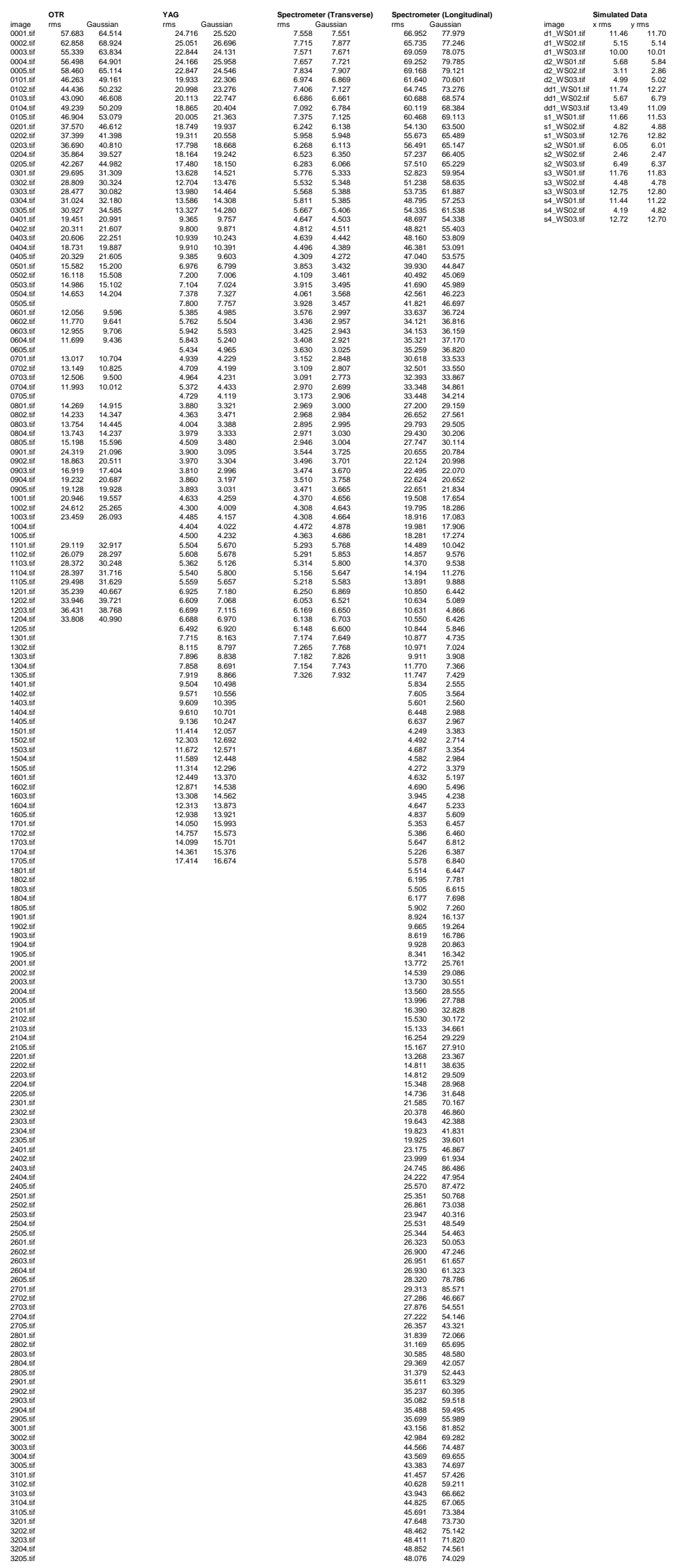
GTF Transverse and Longitudinal Emittance Data Analysis Technique

[1] Transport, SLAC -91, Rev UC-28, 1977. 